\title{
THE USE OF PITUITARY EXTRACTS BY MOUTH IN THE TREATMENT OF DIABETES INSIPIDUS
}

MAURICE H. REES, Ph.D., M.D., AND WM. H. OLMSTED, M.D. Washington University School of Medicine

It has been quite conclusively shown that pituitary extract-posterior lobe-is effective in controlling the polydipsia and polyuria of diabetes insipidus. It has, however, been necessary in practically every case to administer the extract by intramuscular injection. As a rule one injection per day, regardless of the size of the dose, is not sufficient to relieve the patient. Usually two or three injections of $1 \mathrm{cc}$. each are required to keep down both the day and the night intake and output. Since, in most cases, this treatment has to be continued indefinitely a great financial and physical burden must be endured by the patient. The present price of the liquid extract is about 25 cents per 1 cc. ampoule. Since it usually requires three injections per day the cost to the patient would be $\$ 0.75$ per day for the extract, alone. 'To this is to be added the physician's charge for giving the injections, therefore, the cost to the patient could not be less than $\$ 1.50$ per day. Of still greater importance is the patients' loss in time in being required to make three visits per day at the physician's office.

On looking through the literature we find that in practically every reported case of diabetes insipidus the administration of pituitary extracts by mouth has been tried and reported as a failure. In a few cases the feeding of four to seven whole beef glands has been reported as successfully reducing the polyuria during the night.

In the treatment of the following case it was our purpose to find, if possible, a successful method of giving pituitary extract by mouth. Since there is a strong probability that the effective agent of the extract is destroyed in the stomach, we sought to overcome this possible difficulty by giving the extract in salol coated capsules. The preparation used was the desiccated posterior lobe as prepared by Armour \& Co. 
The price of the desiccated posterior lobe extract (Armour $\&$ Co.) is about $\$ 2.00$ per dram. This would make the capsules $(2 \mathrm{gr}$.$) cost at the rate of fifteen for \$ 1.00$. The salol coating would increase this cost slightly. At the rate of four capsules per day the cost to the patient would be $\$ 0.35$ to $\$ 0.50$ per day; about one-third the cost of the injections. The great saving to the patient in time and money is quite evident.

\section{CASE HISTORY}

Case B.V.-The patient was a white male, age 37 , by occupation a railroad brakeman.

His weight at the onset of the present illness was 145 pounds; on admission to the hospital it was 165 pounds; six months after admission to hospital it was 195 pounds.

He was well nourished and well developed, and did not appear to be sick.

He entered Barnes Hospital on March 31, 1921, with the complaint of excessive thirst, frequency of urination, diarrhea and almost constant headache.

His family history was unimportant.

His past history indicated the usual childhood diseases. He had paralysis of right side of face and bilateral impairment of hearing twelve years ago.

Gonorrhea and chancroid, contracted fourteen years ago, received no treatment.

Nine years ago he was troubled with weakness, shortness of breath and nervousness. He lost 30 pounds of weight in one year. $\mathrm{He}$ consulted a physician at this time and was told that he had pulmonary tuberculosis and that he could not live over one and one-half years. At this time the patient began excessive indulgence in alcohol. This excessive drinking was continued for several years.

The patient was married nine years ago and has two children living and well. There is a history of one miscarriage.

His present illness began two years ago and the trouble came on quite rapidly. He first noticed that he was drinking large amounts of water and that he had to get up several times at night to urinate.

Within a month after the patient first noticed his increased thirst and frequency he was voiding large quantities of clear urine about every thirty minutes day and night. He states that on several occasions he measured his urinary output for twenty-four hours and found it to be twenty to twenty-five quarts.

From the beginning of his present trouble the patient has had more or less constant headache. He also complains of dizziness and of being easily fatigued. He thinks that his memory is impaired, and states that his head "feels dead." 
Since the onset of the trouble he has had four or five loose stools per day. His appetite has always been good.

Since one or two months after the onset, the patient's condition has remained quite constant until he entered Barnes Hospital.

Physical examination gave no definite pathological findings.

Neurological examination showed a seemingly normal mental condition. Judgment and memory were good. There were no speech defects, nor pathological reflexes.

Laboratory and special examination showed normal blood findings. The urine showed a very low (1001-1002) specific gravity on admission; the total solids for twenty-four hours were within normal limits.

The blood Wassermann reaction (four taken) was negative. results.

One cerebro-spinal fluid Wassermann was taken with negative

The phenosulphonphthalin test for kidney function showed a return of 75 per cent of the dye. in two hours.

Opthalmoscopic examination showed that the discs were normal. The visual fields were normal.

Stereoscopic plates of the skull showed no definite abnormalities.

A gastric fractional analysis showed a normal acidity curve.

A gastro-intestinal fluoroscopic examination showed a marked hypermotility of the whole tract.

The blood pressure on admission was $110 / 65$; after treatment it was $125 / 70$.

Progress in the Hospital: When the patient was first admitted to our service his twenty-four hour fluid intake and output ranged between twelve to fifteen liters.

In order that we might secure a more stable intake and output we first had the patient control his intake at a point where he could remain for some time with reasonable comfort. This point was found to be about seven liters per twenty-four hours. After thus limiting the intake for three days the urinary output decreased in proportion and the specific gravity went up to 1011 . The patient was now asked still further to reduce his intake. This he did, getting it down, with some difficulty, to slightly less than four liters. The specific gravity went as high as 1013. While on this decreased intake the patient complained of nervousness, dull headache, and sleeplessness.

After keeping the patient on a four liter intake for six days he was put on placebo treatment for four days. The patient thought that this treatment made him less nervous, but it was noted that his intake was gradually increasing.

The patient was now put on 8 minims of adrenalin, t.i.d., by mouth. This failed to control the gradual rise in the intake.

Giving the adrenalin by means of a duodenal tube gave no better results.

The patient was next put on desiccated pituitary extract, posterior lobe (Armour \& Co.), in salol coated capsules. The dose was 
started at one-half grain, t.i.d., and gradually increased to two grains, t.i.d., a.c. and at 10 P. M. The 10 P. M. dose was found to be necessary to control the nocturia.

On the latter treatment the intake came down to between two and a half and three liters and remained there during the two weeks that the treatment was continued. The patient stated that he felt better than he had since the onset of his trouble. His headaches disappeared, he became less nervous. He had no nocturia. The number of stools decreased from 4 or 5 watery stools per day to one or two formed stools per day. The patient stated that he was quite satisfied with two and a half to three liters of fluid per day. His only complaint was some difficulty in getting to sleep at night. This was relieved by giving eight minims of paraldehyde at bedtime.

Before and during the treatment quantitative tests were made on the urine as follows: for urea, uric acid, creatinine and chlorides. Tests were also run on the blood for non-protein nitrogen, urea, uric acid, and creatinine. These constituents were found to be practically normal in amount. There was practically no variation in them after the urine became more concentrated.

During the treatment with pituitary extract the specific gravity of the urine went as high as 1024 on the night specimen and 1019 for the day specimen.

We next tried the effect of intra-muscular injections of pituitary liquid. Single daily injections of 1 c.c. would reduce the output for about twelve hours; but during the next twelve hours there was a marked polyuria so that the total twenty-four hour output was not decreased. Two injections of 1 c.c. each were effective in keeping down the daily output, provided the second injection was given at about ten o'clock at night. Even with two daily injections the intake and output were not reduced below two and one-half liters. This is no greater reduction than was obtained with the dry extract given in salol coated capsules by mouth.

Whenever the patient was taken off all treatment his fluid intake and output increased in two or three days to seven liters or more.

We now tried giving the pituitary extract powder to see whether it would be as effective as when given in salol coated capsules. The powdered extract was given in a small amount of milk in two grain doses, t.i.d., a.c. and at 10 P.M. This method of treatment proved to be quite ineffective in controlling the polydipsia and polyuria. Giving the powdered extract per rectum in four grain doses twice per day gave no results. The patient was discharged from the hospital on June 15,1921 . At this time he was still keeping his intake and output under three liters.

Since leaving the hospital the patient has been unable to secure a sufficient amount of the desiccated extract and he has passed as much as twenty liters of urine per day. 
He also complains of marked fatigue on slight exertion and since leaving the hospital he has been unable to do more than four hours work per day. The fatigue is followed by uncontrollable thirst. The patient feels sure, however, that if he could avoid exertion and could have the pituitary extract regularly, he could control his thirst at any time.

\section{DISCUSSION}

It is possible, in this case, that the marked hypermotility of the alimentary tract may so increase water absorption as to cause the excessive thirst, and incidentally the excessive urinary output. It is also possible that the pituitary extract may decrease the absorption of water either by limiting the intestinal motility or by increasing a local vaso-constriction.

The results obtained in this case would suggest that, in some instances at least, diabetes insipidus may be successfully treated by giving the desiccated posterior lobe substance of the pituitary gland by mouth, provided that it is given in such a way as to pass through the stomach unchanged.

Giving the extract in salol coated capsules seems to give a satisfactory solution to this problem.

\section{Summary}

The possibility of finding a satisfactory method of adminisistering post-pituitary extract by mouth was investigated. Reduced expense and increased convenience for the patient were the principle factors considered.' The history, physical and laboratory findings in a case of diabetes insipidus are given. 'The progress of the patient under various types of treatment is recorded. It was found that by giving desiccated posterior lohe substance in salol-coated capsules the polydipsia an dpolyuria were as effectively controlled as with hypodermic injections of pituitary extract. 